\title{
Nonlinear Optical Response in Single Alkaline Niobate Nanowires
}

\author{
F. Dutto, ${ }^{+, \S}$ C. Raillon, ${ }^{+, \S}$ K. Schenk, ${ }^{\neq}$and A. Radenovic ${ }^{\dagger, *}$ \\ ${ }^{\dagger}$ Laboratory of Nanoscale Biology, Institute of Bioengineering, School of Engineering, EPFL, 1015 Lausanne, École Polytechnique \\ Fédérale de Lausanne (EPFL), CH-1015 Lausanne, Switzerland \\ ${ }^{\ddagger}$ Laboratory for Crystallography, Institute of the Physics of Biological Systems, School of Basic Sciences, EPFL, École Polytechnique \\ Fédérale de Lausanne (EPFL), CH-1015 Lausanne, Switzerland
}

Supporting Information

ABSTRACT: We have synthesized and characterized three types of perovskite alkaline niobate nanowires: $\mathrm{NaNbO}_{3}$, $\mathrm{KNbO}_{3}$, and $\mathrm{LiNbO}_{3}\left(\mathrm{XNbO}_{3}\right)$. All three types of nanowires exhibit strong nonlinear response. Confocal imaging has been employed to quantitatively compare the efficiency of synthesized nanowires to generate second harmonic signal and to show that $\mathrm{LiNbO}_{3}$ nanowires exhibit the strongest nonlinear response. We also investigated the polarization response of the second harmonic generation (SHG) signal in all three types of alkaline nanowires for the two geometries tractable by our
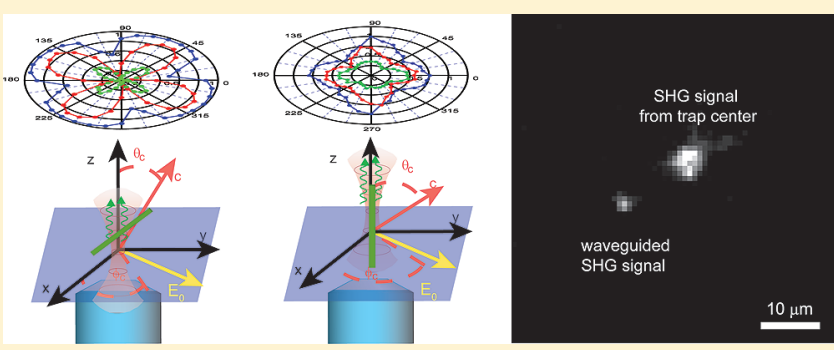
optical trapping setup. The SHG signal is highly influenced by the nanowire crystallinity and experimental geometry. We also demonstrate for the first time wave-guiding of SHG signal in all three types of alkaline niobate nanowires. By carefully examining nonlinear properties of $\left(\mathrm{XNbO}_{3}\right)$ nanowires we suggest which type of wires are best suited for the given application.

KEYWORDS: SHG, optical trapping, synthesis, alkaline nanowires

D iverse applications in nanophotonics require nanostructured active elements that exhibit a strong nonlinear response. Perovskite alkaline niobate nanowires have attracted recently lots of attention having a variety of interesting properties such as significant nonlinear optical response, considerable piezoelectric, pyroelectric, photorefractive, and photocatalytic response $^{1-9}$ as well as superior mechanical and chemical stability. In addition, the latest experiments ${ }^{10}$ demonstrated that these nanostructures can be used as local mechano-optical probes. ${ }^{11,12}$ Although many groups report on the synthesis and structural characterization of different types of alkaline niobate nanowires, there are no comparative studies that address their nonlinear optical response. In this work, we compare SHG signal and waveguiding properties for three types of perovskite alkaline niobate nanowires such as $\mathrm{NaNbO}_{3}, \mathrm{KNbO}_{3}$, and $\mathrm{LiNbO}_{3}\left(\mathrm{XNbO}_{3}\right)$.

To synthesize $\mathrm{XNbO}_{3}$ nanowires, we have used hydrothermal synthesis ${ }^{1,8,9,13-17}$ method. This one step synthesis procedure allows easy variation of different parameters such as concentration, fill factor, time, and oven temperature. This variation results in different synthesized shapes switching from unspecified clusters to cubes or wires. Successful results have been obtained reproducing the $\mathrm{KNbO}_{3}$ recipe described by Wang et al. ${ }^{15,16}$ with single crystalline nanowires of aspect ratio around 20 as shown in Figure $1 \mathrm{~b}$. By following the recipe proposed in recent publication by $\mathrm{Zhu}$ et al. ${ }^{1}$ we have obtained $\mathrm{NaNbO}_{3}$ polycrystalline nanowires having a high aspect ratio up to 50 as shown in Figure 1a. By using X-ray diffraction (XRD) characterization, we confirmed that the $\mathrm{KNbO}_{3}$ nanowires have orthorhombic and cubic structures in percentages of 68 and $32 \%$, respectively, whereas $\mathrm{NaNbO}_{3}$ monoclinic phase has been confirmed in a percentage of $64 \%$ after a calcination step ${ }^{14,18}$ (see Supporting Information Figures $2 \mathrm{c}$ and $3 \mathrm{c}$ ). We tried to vary growth parameters during hydrothermal synthesis of $\mathrm{LiNbO}_{3}$ nanowires, but it appears that this method is not best suited for production of this material. ${ }^{8}$

The failure of this synthetic route might arise from the lower solubility limit of $\mathrm{LiOH}(12.8 \mathrm{~g} / 100 \mathrm{~mL})$ compared to $\mathrm{KOH}$ $(110 \mathrm{~g} / 100 \mathrm{~mL})$ and $\mathrm{NaOH}(111 \mathrm{~g} / 100 \mathrm{~mL})$, resulting in a difficult mixing of the chemicals due to $\mathrm{LiOH}$ precipitation. While trying to synthesize $\mathrm{LiNbO}_{3}$ nanowires, we regularly checked the obtained compound by XRD and our results point to an absence of desired $\mathrm{LiNbO}_{3}$ nanowires but instead indicate only presence of $\mathrm{Nb}_{2} \mathrm{O}_{5}$, the raw precursor material (data not shown). Since the synthesis of $\mathrm{LiNbO}_{3}$ nanowires via hydrothermal method has been clearly unsuccessful, we proceeded by following the molten salt synthesis reported recently by Santulli et al. ${ }^{19,20}$ and were able to grow single crystalline $\mathrm{LiNbO}_{3}$ wires with an average aspect ratio of 10 (see Figure 1c).

Together with bulk XRD measurements we also performed TEM characterization of single $\mathrm{XNbO}_{3}$ nanowires, and when operating in the diffraction mode we took selected area electron diffraction (SAED) patterns. Those patterns taken on the single $\mathrm{XNbO}_{3}$ nanowires are useful to determine the degree of structural order in a single nanowire and to confirm the crystal structure

Received: March 31, 2011

Revised: $\quad$ May 6, 2011

Published: May 24, 2011 

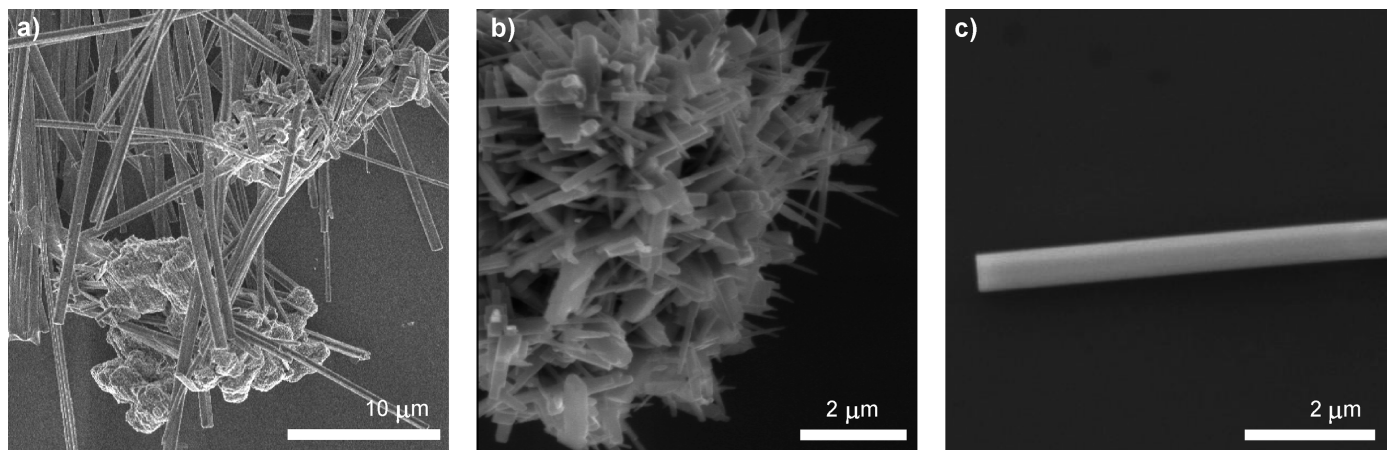

Figure 1. (a) SEM images of hydrothermally synthesized $\mathrm{NaNbO}_{3}$ nanowires and (b) KNbO3 nanowires. (c) SEM image of molten salt synthesized $\mathrm{LiNbO}_{3}$ nanowire.
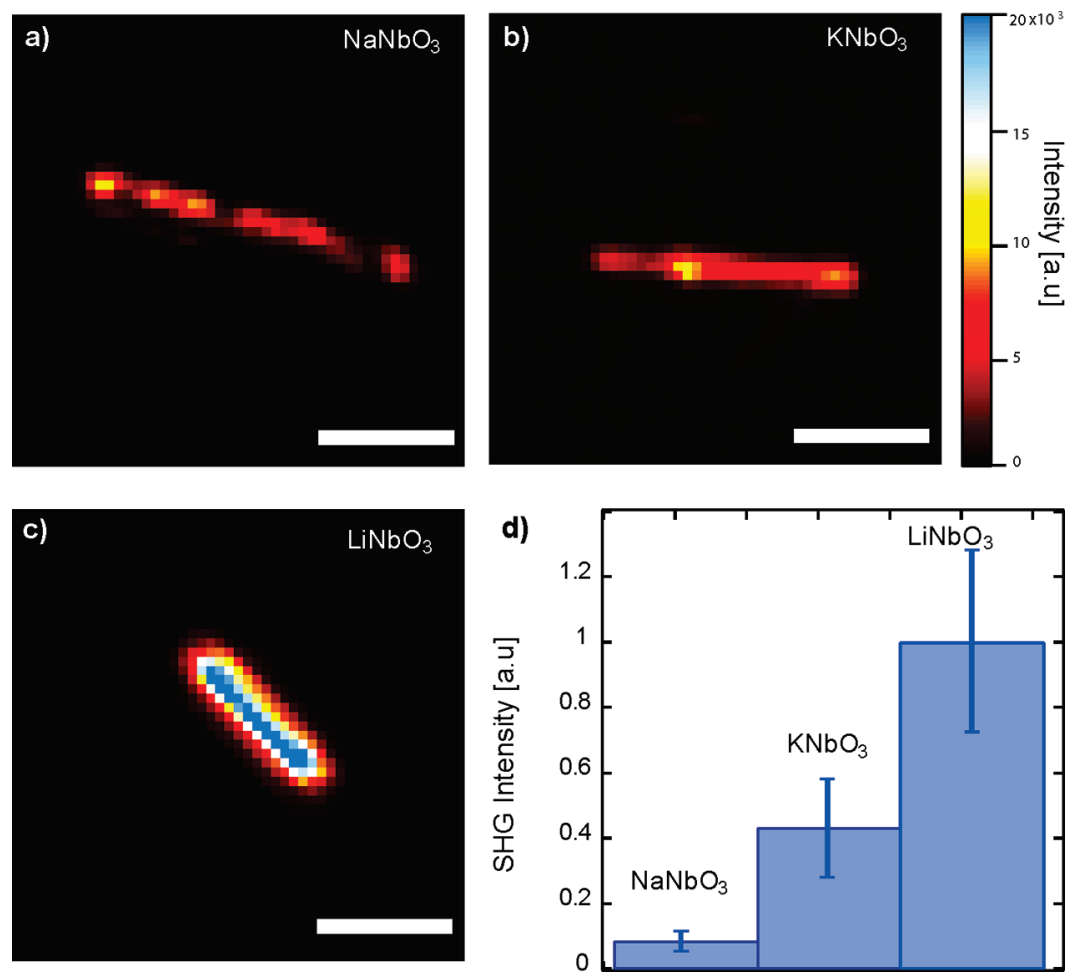

Figure 2. Confocal images of (a) hydrothermally synthesized $\mathrm{NaNbO}_{3}$ nanowire and (b) $\mathrm{KNbO}_{3}$ nanowire. (c) Confocal image of molten salt synthesized $\mathrm{LiNbO}_{3}$ nanowire. (d) Average, volume scaled, $\mathrm{SHG}$ signal for each type of $\mathrm{XNbO}_{3}$ nanowires. The error bar shows the standard deviation of the volume scaled SHG signal for each nanowire type. The scale bar is $3 \mu \mathrm{m}$.

obtained with XRD measurement. Our results (Supporting Information Figures 2, 3, and 4) confirmed a high crystallinity of $\mathrm{KNbO}_{3}$ and $\mathrm{LiNbO}_{3}$ nanowires and lower crystallinity of $\mathrm{NaNbO}_{3}$ nanowires. The samples used for SEM, TEM, and SAED characterization were dispersed in absolute ethanol and ultrasonicated prior to deposition onto TEM or SEM compatible substrates.

Having successfully produced three types of perovskite $\mathrm{XNbO}_{3}$ nanowires, namely $\mathrm{NaNbO}_{3}, \mathrm{KNbO}_{3}$, and $\mathrm{LiNbO}_{3}$, we decided to examine and compare their nonlinear optical response. We first used a standard scanning confocal microscope (Leica SP5) for testing whether our synthesized wires generated any SHG signal. This approach permits a fast characterization of many nanowires. Unlike fluorescence, the SHG process occurs without nonradiative energy loss and involves only virtual electron energy transition; therefore SHG structures do not bleach over time and emit a stable, nonblinking signal that does not saturate with increasing excitation power. Furthermore, SHG is generally a nonresonant process that offers flexibility in the choice of excitation wavelength. This also results in the flexibility of tuning the wavelength of the SHG signal by changing the excitation wavelength accordingly.

Prior to imaging, nanowires were deposited on a glass slide and then observed on the confocal microscope. The excitation light source consisted of linearly polarized femtosecond laser pulses (Coherent, Chameleon) and the excitation wavelength was centered at $854 \mathrm{~nm}$ and delivered to the sample through $63 \times$ 1.2 NA water immersion objective. The SHG signal was detected by a photomultiplier preceded by an optical bandpass filter centered at $427 \mathrm{~nm}$ (having a detection width of $20 \mathrm{~nm} \mathrm{417-}$ $437 \mathrm{~nm}$ ). In order to compare nonlinear optical response from all 

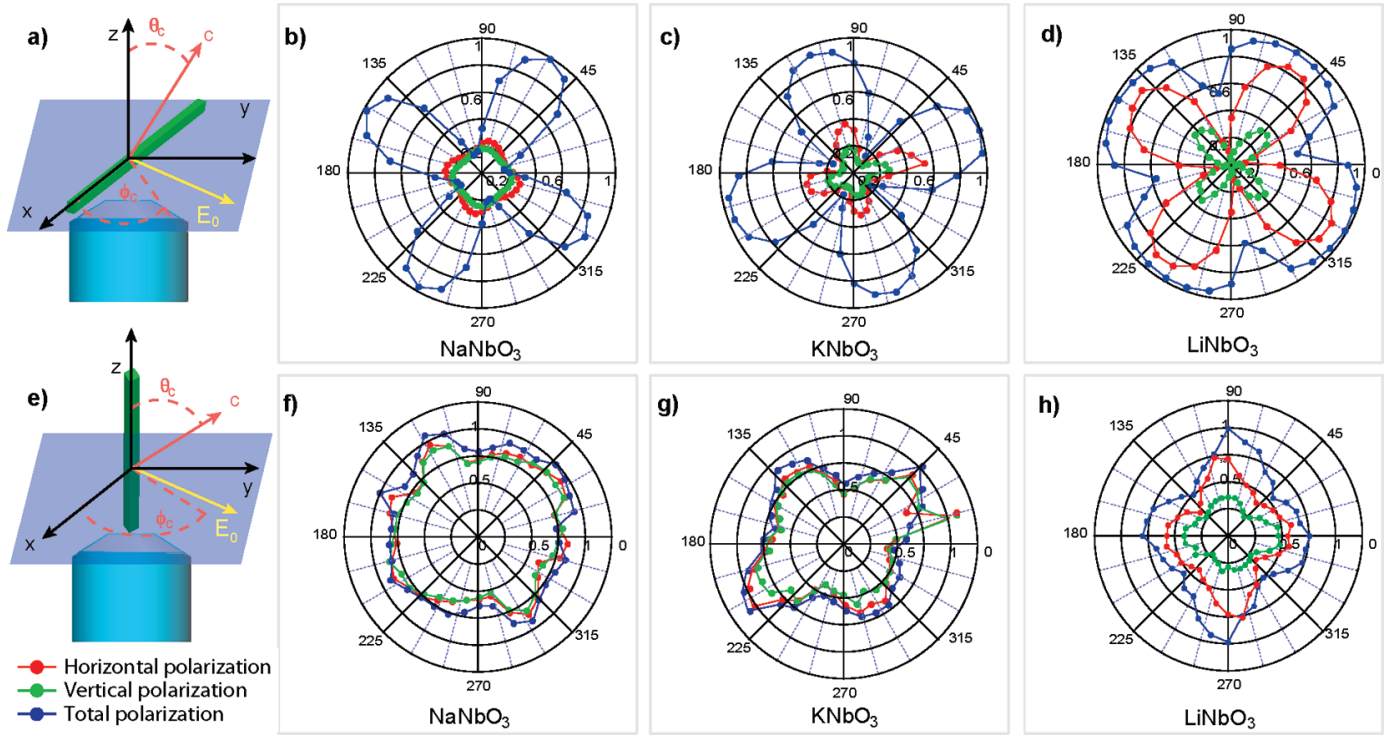

Figure 3. (a) Schematics of experimental geometry employed for polarization experiments on the stuck nanowires, where $\theta_{\mathrm{c}}$ indicates an angle of inclination of crystal $c$-axis with respect to the nanowire geometric axis and $\phi_{c}$ an azimuth of the incident linearly polarized light. (b) Polar plot of the normalized SHG signal from the stuck $\mathrm{NaNbO}_{3}$ nanowire for three experimental conditions. (c) Polar plot of the normalized SHG signal from the stuck $\mathrm{KNbO}_{3}$ nanowire for three experimental conditions. (d) Polar plot of the normalized SHG signal from the stuck $\mathrm{LiNbO}_{3}$ nanowire for three experimental conditions. (e) Schematics of experimental geometry employed for polarization experiments on the optically trapped nanowires. (f) Polar plot of the normalized SHG signal from the optically trapped $\mathrm{NaNbO}_{3}$ nanowire for three experimental conditions. g) Polar plot of the normalized SHG signal from the optically trapped $\mathrm{KNbO}_{3}$ nanowire for three experimental conditions. h) Polar plot of the normalized SHG signal from the optically trapped $\mathrm{LiNbO}_{3}$ nanowire for three experimental conditions.

three types of nanowires, we kept the excitation power set to $3 \mathrm{~mW}$ and displayed $\mathrm{SHG}$ signals from each type of single $\mathrm{XNbO}_{3}$ nanowire at the same scale as shown in Figure $2 \mathrm{a}-\mathrm{c}$. It is evident from Figure 2 that $\mathrm{LiNbO}_{3}$ displays the highest nonlinear response. To address possible variation in SHG signal due to wire to wire crystallinity dissimilarities and the fact that SHG signal scales with the wire dimension, we have collected SHG images of more than 20 nanowires for each type of $\mathrm{XNbO}_{3}$ nanowires, integrated total SHG per each wire, and scaled it with the corresponding wire volume. Volume for each wire was obtained from bright field and SEM images. For the details see Supporting Information Figure 5 and Supporting Information. This allows us to compute the average SHG signal for all three nanowire materials. Data in Figure $2 \mathrm{~d}$ summarize our results, confirming the strongest nonlinear response from $\mathrm{LiNbO}_{3}$ nanowires. Our results obtained for the nanowires are in good agreement with the reported bulk values for alkaline niobate materials. $^{18,21-26}$

After comparing intensity of SHG signal in the synthesized nanowires, we decided to investigate how the integral intensity and the polarization of SHG signals depend on the impinging light polarization. In this paper, the second harmonic signal is either generated in optically trapped nanowires or in nanowires stuck to the glass substrate. In the optically trapped nanowires (for $\theta_{c}=0$ ) the nanowire optical $c$-axis lies along the light propagation ( $z$-axis of our coordinate system) while for the stuck nanowire it is perpendicular to it. Both experimental geometries are shown in Figure 3a,e. The sample cell is prepared by transferring a solution of water-dissolved nanowires into a chamber created with $120 \mu \mathrm{m}$ thick double-sided tape (Grace Biolabs) between a glass slide and a glass coverslip. Once sealed inside the chamber, the nanowires sank toward the bottom. One fraction of the wires is usually already stuck to the glass coverslip, while the rest of the nanowires and nanowire clusters undergo Brownian motion allowing us to easily test both geometries using the same setup and same sample cell (details regarding the setup can be found in Supporting Information). When the laser is brought close to a nanowire, it is pulled into the trap, ${ }^{11}$ trapped tridimensionally perpendicular to the surface and the SHG signal can be detected by the EMCCD camera. Varying the polarization of the impinging light, by moving a half wave plate the reliance of the SHG signal on light polarization is studied. The half wave plate position is controlled by computer, moving a motor installed on the polarizer. In order to perform efficiently this kind of measurement, a LabVIEW program has been written in which both the EMCCD camera and the polarizer can be controlled simultaneously (for details on experimental conditions see Supporting Information). While varying the excitation beam polarization, polarization of the light emitted from the nanowire was analyzed. We have collected data without analyzer in front of the EMCCD and by setting the analyzer to 0 and $90^{\circ}$ corresponding to total, horizontal, and vertical polarization as shown in Figure 3. The polarizer was rotated $10^{\circ}$ between each measurement through a range of $2 \pi$ radians. The polarization change is found to depend sensitively on the nanowire type, geometrical shape, orientation of the nanowire $c$-axis and employed experiment geometry. In all experiments, we used a $1064 \mathrm{~nm}$ CW laser with $100 \mathrm{~mW}$ laser power; in both geometries and for all three types of nanowires, the delivered power generated signal was in the range $(10-40) \mathrm{nW}$. Although this experimental yields more information regarding nonlinear properties of single $\mathrm{XNbO}_{3}$ nanowires, it is unpractical for quantitative comparative studies between different materials, since the SHG signal does not depend solely on the material properties and $c$ axis orientation but it also scales with nanowire dimensions. 

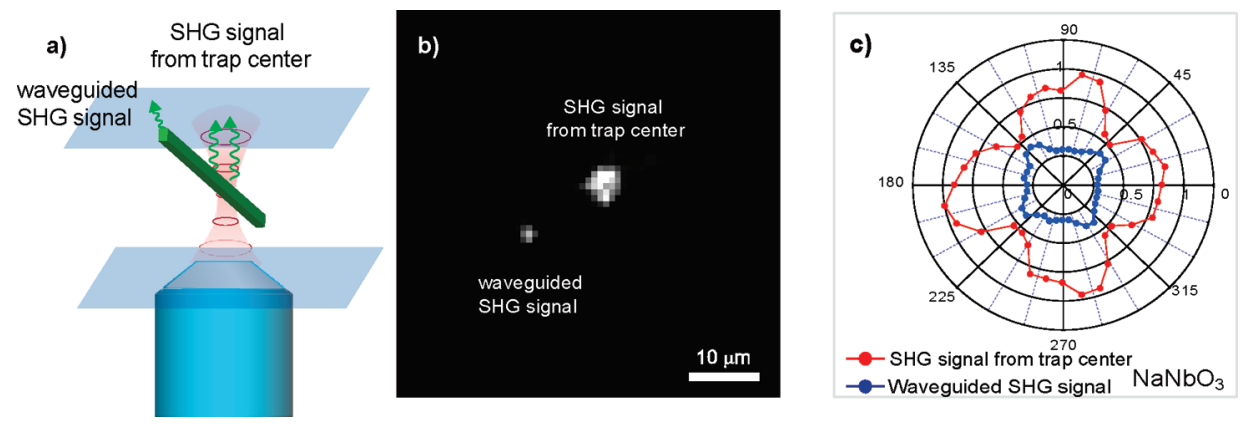

Figure 4. (a) Schematics of experimental geometry employed for wave-guiding experiments. (b) SHG image of the trapped nanowire; its docked end clearly waveguides SHG photons. (c) Polar plot of the normalized SHG signal and wave-guided SHG signal.

Nevertheless these experimental geometries allowed us to investigate how the SHG signal depends on the impinging light polarization. For stuck $\mathrm{KNbO}_{3}$ and $\mathrm{LiNbO}_{3}$ nanowires, we observe strong dependence of the generated SHG see Figure $3 \mathrm{c}, \mathrm{d}$ while for $\mathrm{NaNbO}_{3}$ nanowires this dependence is much lower. We attribute it to the much lower crystallinity of synthesized $\mathrm{NaNbO}_{3}$ nanowires in respect to $\mathrm{KNbO}_{3}$ and $\mathrm{LiNbO}_{3}$. This is confirmed from our XRD measurements and TEM characterization of synthesized nanowires. As already suggested by Grange et al., ${ }^{8}$ the polarization dependence of SHG signal might be used as an easy way to evaluate the degree of structural order and the $c$-axis orientation in synthesized nanostructures. We assumed that in optically trapped nanowires we would observe similar dependence. As shown in Figure $3 \mathrm{f}-\mathrm{h}$ the dependence is probably masked by the Brownian motion of the trapped nanowire. ${ }^{27}$ To investigate whether nanowires confined in the stiffer trap exhibit higher dependence upon the impinging light polarization, we have trapped the $\mathrm{KNbO}_{3}$ at two laser power as shown in Supporting Information Figure 6, and for both powers we collected SHG signal as a function of the impinging light polarization. We observe, as expected, that the signal obtained from the stiffer trap displays higher symmetry. Occasionally, we noticed that some wires would oscillate due to the generation of net nonzero torque by the trap. We have also noticed that the trapping of long $\mathrm{LiNbO}_{3}$ nanowires that generate enough signal is much less stable in comparison to short $\mathrm{LiNbO}_{3}$ nanoparticles. This problem probably arises from the specific nanowires shape. The presented method indicates that the strength of the SHG signal can be easily optimized by proper orientation of the impinging light polarization in respect to the nanowire $c$-axis orientation.

Although $\mathrm{XNbO}_{3}$ nanowires should be excellent low loss subwavelength waveguides, so far only one study addressed their wave-guiding prosperities. ${ }^{28}$ In addition, wave-guiding of SHG signal has been observed only in $\mathrm{KNbO}_{3}$ in the experiment where the wave-guided SHG signal excites the fluorescence on the bead $^{12}$ allowing long acquisition time. In this work, we present for the first time direct evidence that all three types of $\mathrm{XNbO}_{3}$ nanowires can efficiently waveguide the SHG signal (see Figure 4). To demonstrate it, we have selected for trapping only wires that are longer than $10 \mu \mathrm{m}$. After successful trapping of one nanowire end, the wire has been brought in close contact with the glass coverslip (see Figure 4a) allowing unmasked observation of wave-guided SHG signal. In addition, short acquisition time allowed, as in the previous experiment, to change the impinging laser polarization (Supporting Information Movies 1-3). We believe that the large refractive index (for all three types of nanowires, it ranges form 2.02-2.4) provides tight optical confinement for low-order modes and also results in a large acceptance angle for coupling light into the nanowire waveguide, resulting in the relatively high in-coupling efficiency up to $80 \%$ (see Figure 4c).

In summary, we report several interesting points. We have performed comparative studies of $\mathrm{SH}$ signal in $\mathrm{XNbO}_{3}$ nanowires and established the largest nonlinear response in $\mathrm{LiNbO}_{3}$. In addition, we show that the intensity of $\mathrm{SH}$ signal is polarization dependent and a function of the degree of structural order in synthesized nanowires. For single crystalline $\mathrm{XNbO}_{3}$ nanowires, the resulting $\mathrm{SH}$ intensity change could be used for monitoring the rotational movement of biological samples. Efficient waveguiding of the $\mathrm{SH}$ signal through nanowires is prerequisite for a future use of the $\mathrm{XNbO}_{3}$ nanowires as local noninvasive light sources into biological cells or tissues. On the basis of our nanowire characterization, we can associate the following application for each type of $\mathrm{XNbO}_{3}$ nanowires: as $\mathrm{LiNbO}_{3}$ nanowires display highest nonlinear response they are best suited for the use as markers in imaging. On the other hand, their trapping stability is much worse compared to $\mathrm{KNbO}_{3}$ or $\mathrm{NaNbO}_{3}$ therefore as opto-mechanical probes we suggest to use $\mathrm{KNbO}_{3}$ due to their good trapping stability and relatively high nonlinear response. As all three types of $\mathrm{XNbO}_{3}$ nanowires display similar in-coupling wave-guiding efficiency as potential waveguides, we would recommend $\mathrm{NaNbO}_{3}$ nanowires due to their highest aspect ratio.

To conclude, more research remains for investigating methods to increase $\mathrm{SH}$ signal conversion efficiency in $\mathrm{XNbO}_{3}$ nanowires or their practical functionality in photonic circuits. Furthermore, their bimolecular functionalization is crucial for their use in biomedical imaging.

\section{ASSOCIATED CONTENT}

S Supporting Information. Supporting Information containing Supplementary Methods with legends and additional references, supplementary Figures $1-7$ and Supplementary Movies $1-3$. This material is available free of charge via the Internet at http://pubs.acs.org.

\section{AUTHOR INFORMATION}

\section{Corresponding Author}

*E-mail: aleksandra.radenovic@epfl.ch.

\section{Author Contributions}

$\S$ These authors contributed equally. 


\section{ACKNOWLEDGMENT}

This work was supported by Swiss National Science Foundation (FNS) with Grants 200021-125319 and CRSII3_132396. We thank the Centre Interdisciplinaire de Microscopic Electronique (CIME) at EPFL for access to electron microscopes and Bioimaging and Optics Platform (PTBIOP) for access to confocal microscope. A.R., C.R., and F.D. designed the study. F.D. synthesized the samples and performed SHG measurements presented in the paper and Supporting Information. F.D. with the help of C.R. carried out TEM and SEM sample characterization. K.S. performed bulk powder X-ray diffraction measurements. C.R and A.R. built optical trapping setup. F.D. and A.R. analyzed data presented in the paper and Supporting Information and prepared the manuscript. All the authors read and commented on the manuscript.

\section{REFERENCES}

(1) Zhu, H. Y.; Zheng, Z. F.; Gao, X. P.; Huang, Y. N.; Yan, Z. M.; Zou, J.; Yin, H. M.; Zou, Q. D.; Kable, S. H.; Zhao, J. C.; Xi, Y. F.; Martens, W. N.; Frost, R. L. J. Am. Chem. Soc. 2006, 128 (7), 2373-2384.

(2) Jehng, J. M.; Wachs, I. E. J. Raman Spectrosc. 1991, 22 (2), 83-89.

(3) Lin, C. H.; Lee, C. H.; Chao, J. H.; Huang, Y. M.; Chang, H. W.; Kuo, C. Y.; Hsu, C. W. Mater. Chem. Phys. 2005, 92 (1), 128-133.

(4) Goh, G. K. L.; Lange, F. F.; Haile, S. M.; Levi, C. G. J. Mater. Res. 2003, 18 (2), 338-345.

(5) Santos, I. C. M. S.; Loureiro, L. H.; Silva, M. F. P.; Cavaleiro, A. M. V. Polyhedron 2002, 21 (20), 2009-2015.

(6) Cho, C. R. Mater. Lett. 2002, 57 (4), 781-786.

(7) Nowak, I.; Ziolek, M. Chem. Rev. 1999, 99 (12), 3603-3624.

(8) Grange, R.; Choi, J. W.; Hsieh, C. L.; Pu, Y.; Magrez, A.; Smajda, R.; Forro, L.; Psaltis, D. Appl. Phys. Lett. 2009, 95, 143105.

(9) Magrez, A.; Vasco, E.; Seo, J. W.; Dieker, C.; Setter, N.; Forro, L. J. Phys. Chem. B 2006, 110 (1), 58-61.

(10) Law, M.; Sirbuly, D. J.; Johnson, J. C.; Goldberger, J.; Saykally, R. J.; Yang, P. D. Science 2004, 305 (5688), 1269-1273.

(11) Pauzauskie, P. J.; Radenovic, A.; Trepagnier, E.; Shroff, H.; Yang, P. D.; Liphardt, J. Nat. Mater. 2006, 5 (2), 97-101.

(12) Nakayama, Y.; Pauzauskie, P. J.; Radenovic, A.; Onorato, R. M.; Saykally, R. J.; Liphardt, J.; Yang, P. D. Nature 2007, 447 (7148), 1098-U8.

(13) An, C. H.; Tang, K. B.; Wang, C. R.; Shen, G. Z.; Jin, Y.; Qian, Y. T. Mater. Res. Bull. 2002, 37 (11), 1791-1796.

(14) Shi, H. F.; Li, X. K.; Wang, D. F.; Yuan, Y. P.; Zou, Z. G.; Ye, J. H. Catal. Lett. 2009, 132 (1-2), 205-212.

(15) Wang, G. Z.; Selbach, S. M.; Yu, Y. D.; Zhang, X. T.; Grande, T.; Einarsrud, M. A. CrystEngComm 2009, 11 (9), 1958-1963.

(16) Wang, G. Z.; Yu, Y. D.; Grande, T.; Einarsrud, M. A. J. Nanosci. Nanotechnol. 2009, 9 (2), 1465-1469.

(17) Wu, S. Y.; Liu, X. Q.; Chen, X. M. Ceram. Int. 2010, 36 (3), 871-877.

(18) Ke, T. Y.; Chen, H. A.; Sheu, H. S.; Yeh, J. W.; Lin, H. N.; Lee, C. Y.; Chiu, H. T. J. Phys. Chem. C 2008, 112 (24), 8827-8831.

(19) Santulli, A. C.; Zhou, H. J.; Berweger, S.; Raschke, M. B.; Sutter, E.; Wong, S. S. CrystEngComm 2010, 12 (10), 2675-2678.

(20) Li, L. H.; Deng, J. X.; Chen, J.; Sun, X. Y.; Yu, R. B.; Liu, G. R.; Xing, X. R. Chem. Mater. 2009, 21 (7), 1207-1213.

(21) Sutherland, R. L. Handbook of nonlinear optics; Marcel Dekker: New York, 1996; p 685.

(22) Fluck, D.; Gunter, P. IEEE J. Sel. Top. Quantum Electron. 2000, $6(1), 122-131$.

(23) Shoji, I.; Kondo, T.; Ito, R. Opt. Quantum Electron. 2002, 34 (8), 797-833.

(24) Weber, M. J. Handbook of optical materials; CRC Press: Boca Raton, 2003; p 512.

(25) Boyd, R. W. Nonlinear optics, 3rd ed.; Academic Press: Amsterdam, 2008; p xix, 613.
(26) Johnston, K. E.; Tang, C. C.; Parker, J. E.; Knight, K. S.; Lightfoot, P.; Ashbrook, S. E. J. Am. Chem. Soc. 2010, 132 (25), 8732-8746.

(27) Bareil, P. B.; Sheng, Y. L. Optical Trapping and Optical Micromanipulation Vii 2010, 7762, 605.

(28) Huang, J.; Chen, Z.; Zhang, Z.; Zhu, C.; He, H.; Ye, Z.; Qu, G.; Tong, L. Appl. Phys. Lett. 2011, 98 (9), 093102. 